\title{
In-situ Observations of the Complex Crystallization Processes Occurring Laser Heated Amorphous Germanium Films
}

Thomas LaGrange,* Liliya Nikolova, ** Mark Wall, *Bryan W. Reed,* Geoffrey H. Campbell, Bradley J. Siwick, *** and Federico Rosei**

* Lawrence Livermore National Laboratory, Physical and Life Science Directorate, Condensed Matter and Materials Division, 7000 East Avenue, P.O. 808 L-356

** Institut National de la Recherche Scientifique Center Energy Materials Telecommunications, 1650 boul. Lionel-Boulet, Varennes, QC, J3X 1S2, Canada

*** Departments of Physics and Chemistry, Center for the Physics of Materials, McGill University, 801 Sherbrooke St. W., Montreal, QC, H3A 2K6, Canada

The crystallization of amorphous Ge films induced by laser [1,2] heating is quite complex, creating an exotic microstructure that forms over time scales ranging from a few nanoseconds to several microseconds after pulsed laser excitation (see Figure 1). High quality information on the final postmortem structure can be obtained with a variety of techniques. However, structural characterization of short-lived nonequilibrium states has shown to be very challenging. Revealing the dynamics of the structural transformations in materials requires direct observations with at most nanosecond temporal resolution with a spatial resolution of few nanometers [3]. The recently developed dynamic transmission electron microscope (DTEM) at LLNL permits in situ observation of laser induced crystallization behavior with $\sim 10 \mathrm{~nm}$ spatial resolution and $15 \mathrm{~ns}$ temporal resolution [3].

We used the single-shot nanosecond imaging capabilities of the DTEM to capture the complex crystallization behavior of a-Ge films. During crystallization, three distinct microstructural regions form, (1) nanocrystalline region, (2) radial dendritic-like grains, and (3) azimuthally orientated grains (see Figure 1). Crystallization initiates at times less than 20ns and zone (1) fully crystallizes within $55 \mathrm{~ns}$ (see reference [2] for details). The large dendritric grains emerge from the nanocrystalline zone that act as nuclei and grow at rates exceeding $10 \mathrm{~m} / \mathrm{s}$ to lengths of several microns (see the time resolved images in Figure 2). After delays longer than $1 \mu$ s from the laser heating pulse, the growth mode at the periphery of the laser irradiated zone changes from radial along the direction heat diffusion to tangential, perpendicular to the thermal gradient, forming zone (3) that consist of "azimuthally orientated" layers comprised of large faceted crystals (white contrast) and nanocrystals (dark grey). The time-resolved images in Figure 3 clearly show this tangential growth mode that develops over a period of $\sim 10 \mu \mathrm{s}$. The complexity of the growth dynamics reveal the underlying importance of interface energy on the crystallization kinetics. To discern the influence of this anisotropic growth behavior on the crystallization process, we have studied the local crystallography, post mortem in the TEM, using the novel NanoMEGAS ASTAR automated orientation imaging tool. In general, the dendritic grains exhibit a weak $<100>$ fiber texture common for dendetric growth in cubic systems and are highly faceted containing many twin defects (see Figure 4). This presentation will discuss the crystallography of laser devitrified Ge films in terms of the crystallization mechanisms elucidated by the time-resolved studies using the DTEM.

References:

[1] O. Bostanjoglo, W. Marine, P. Thomsen-Schmidt, Appl. Surf. Sci. 54, 302 (1992)

[2] L. Nikolova, T. LaGrange, B. W. Reed, M. J. Stern, N. D. Browning, G. H. Campbell, J. C. 
Kieffer, B. J. Siwick, and F. Rosei, Appl.

Phys. Lett. 97, (2010).

[3] T. LaGrange, G.H. Campbell, B.W. Reed, M. Taheri, J.B. Pesavento, J.S. Kim, N.D. Browning, Ultramicroscopy 108, 1441 (2008)

[4] Work preformed at LLNL under the auspices of the U.S. Department of Energy by Lawrence Livermore National Laboratory under Contract DE-AC52-07NA27344 and supported in part by the U.S. Department of Energy, Office of Basic Energy Sciences, Division of Materials Sciences and

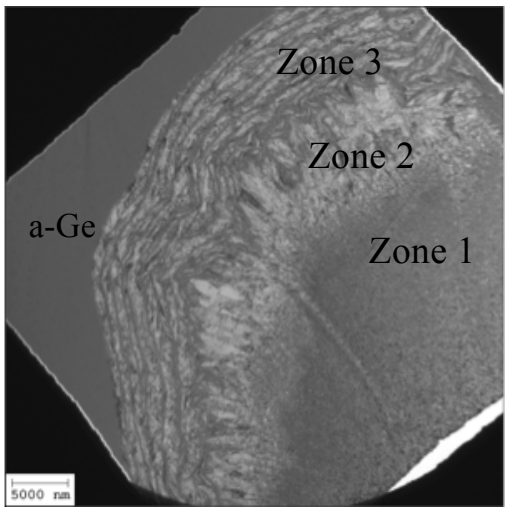

FIG 1. Low magnification micrograph of the lasercrystallized region $(90 \mu \mathrm{m}$ in diameter). Engineering.

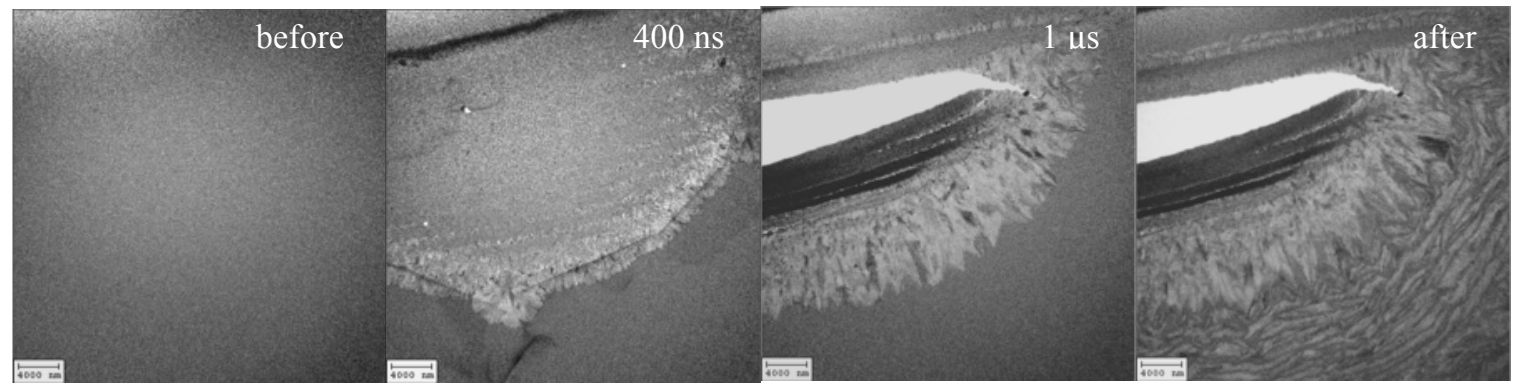

FIG 2. Time-resolved images with a $15 \mathrm{~ns}$ exposure time showing the growth dynamics of the radial, dendritic grains in Zone (2) at two different time delays, $400 \mathrm{~ns}$ and $1 \mu \mathrm{s}$.

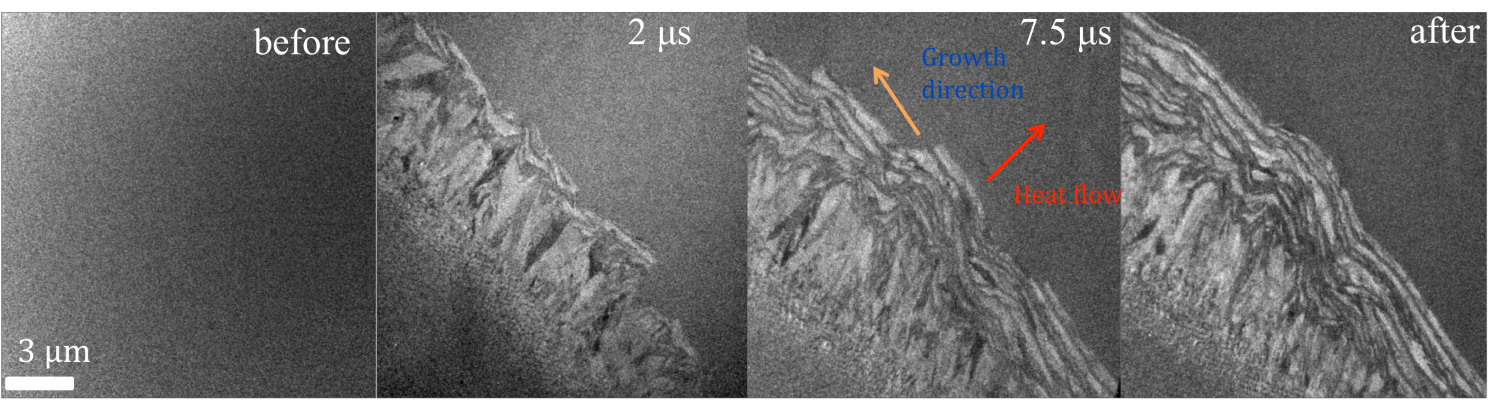

FIG 3. Time-resolved images with a $15 \mathrm{~ns}$ exposure time showing the growth dynamics of the "azimuthally" orientated layers in Zone (3) that grow perpendicular to the heat flow.

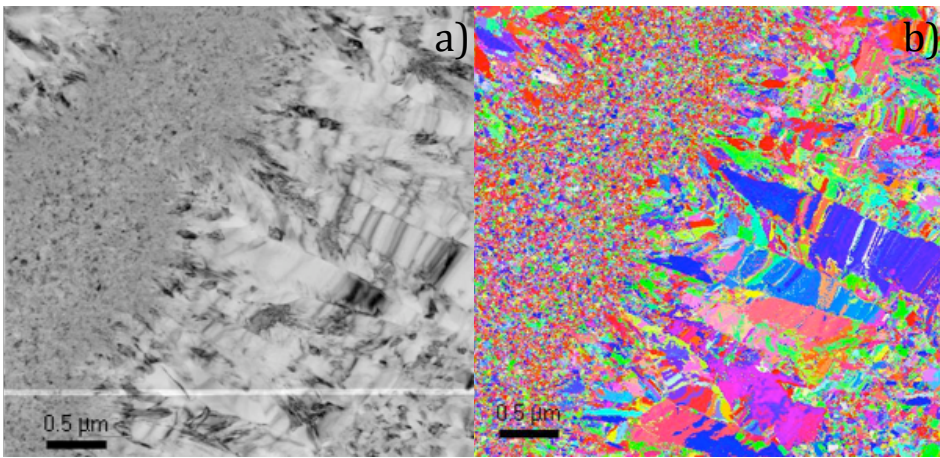

FIG 4. Post mortem images of the laser crystallized microstructure at interface between Zone (1) and (2) taken using the NanoMEGAS, inc. ASTAR ${ }^{\circledR}$ automated orientation mapping system, a) virtual brightfield, b) crystallographic orientation map of the same region. 